\title{
Larval Food Supply Constrains Female Reproductive Schedules in Hippodamia convergens (Coleoptera: Coccinellidae)
}

\author{
GERMAN VARGAS, ${ }^{1}$ J. P. MICHAUD,${ }^{1,2}$ AND JAMES R. NECHOLS ${ }^{3}$
}

\begin{abstract}
Ann. Entomol. Soc. Am. 105(6): 832-839 (2012); DOI: http://dx.doi.org/10.1603/AN12010
ABSTRACT Reproductive schedules are a critical aspect of life history intrinsically linked to a species' ecology. We explored dynamic trajectories of daily fecundity, egg size, and egg fertility in three size classes of Hippodamia convergens Guerin-Meneville produced by varying larval access to food, eggs of Ephestia kuehniella Zeller. Adult pairs were held with ad libitum food and eggs were collected daily, counted, and a subsample measured. Egg fertility declined steeply over 25 clutches in small females, gradually in large females, but remained relatively stable in medium females. In small females, egg size and daily fecundity declined in a linear manner. There was no clear indication of an egg size-number tradeoff. In medium females, both egg size and daily fecundity peaked around the 16th day of oviposition, after which both declined. Large females began oviposition earlier and achieved peak egg size about day 7 , and peak fecundity around day 12. Large females thus expressed a larger proportion of their reproductive effort early in adult life, a strategy inferred to be adaptive in the context of aphidophagy; a larger proportion of progeny would develop early in the exponential growth phase of the prey population when food is most abundant. Increases in egg size during this period may improve the survival of later-developing progeny; prey become scarce as aphid outbreaks decline and competition intensifies, favoring offspring with a larger body size at eclosion. Larval diet restriction appeared to constrain these presumably adaptive changes in egg size and daily fecundity, largely through effects on maternal body size.
\end{abstract}

KEY WORDS aphidophagy, egg size, fecundity, maternal effect, reproductive effort

Most species in the family Coccinellidae are predaceous on other insects or mites (Hodek 1996) and many are relatively specialized predators of aphids. Because of the 'boom and bust' nature of aphid outbreaks and their ephemeral occurrence, aphidophagy is an ecological specialization that selects for rapid development, high fecundity, and a relatively fast pace of life (Dixon 2000, Borges et al. 2006). Because aphidophagous larvae must feed during the exponential growth phase of the aphid population to have sufficient food to complete development, females must oviposit within a short period, the 'oviposition window' to maximize the survival of their progeny (Kindlmann and Dixon 1993, 2011). Thus, females exploiting an aphid outbreak face a predictable trajectory of change in habitat quality for their offspring; over a time frame as short as 2 or $3 \mathrm{wk}$; initially abundant prey become increasingly scarce and competition and intraguild predation intensify. Consequently, aphidophagous coccinellids typically exhibit a triangular fecundity function (Dixon and Agarwala 2002, Michaud and Qureshi 2006) and lay as many eggs as possible early in adult life. The current study used

\footnotetext{
${ }^{1}$ Department of Entomology, Kansas State University, Agricultural Research Center-Hays, 1232 240th Ave., Hays, KS, 67601.

${ }^{2}$ Correspondence author, email: jpmi@ksu.edu.

${ }^{3}$ Department of Entomology, Kansas State University, 123 W. Waters Hall, Manhattan KS, 66506.
}

the convergent lady beetle, Hippodamia convergens Guerin-Meneville, to explore whether this trajectory of reproductive effort (RE) is constrained by female body size, and whether any changes in egg size are associated with changes in clutch size.

The concept of tradeoffs in the allocation of resources to biological processes such as reproduction is central to life history theory (Stearns 1989, Roff 1992). Iteroparous organisms engage in multiple bouts of reproduction over the course of their adult lives and must allocate some proportion of their total RE to each bout, or clutch. To some degree, current investments curtail future reproductive opportunities; the 'cost of reproduction' (Williams 1966, Bell and Koufopanou 1986). Furthermore, within each reproductive bout, females face a tradeoff between producing many small propagules, or fewer large ones (Williams 1966, Smith and Fretwell 1974, Brockelman 1975). Although egg size is often correlated with offspring survival and rate of development, it is shaped by selection acting on maternal fitness, rather than the fitness of the offspring (Roff 1992, Stearns 1992). Because maternal fitness is determined by offspring number, one might expect females to produce eggs close to the minimum size necessary for offspring survival and maximize clutch size to the extent permitted by available resources (Einum and Fleming 2000). This reasoning leads to the general expectation that clutch size will vary more 
than egg size, and that larger females should produce more eggs rather than larger ones. These predictions are largely supported for highly fecund species that provide little or no parental care, that is, most arthropods in general, and coccinellids in particular (Stewart et al. 1991, Dixon and Guo 1993, Carriere and Roff 1995, Dixon 2000, Fox and Czesak 2000). However, the minimal egg size necessary to ensure offspring survival may vary with ecological conditions, or change over the reproductive life of a female, yielding scenarios that require phenotypic plasticity in egg size to maximize maternal fitness. Thus, objections have arisen to static optimization models of egg size (e.g., Charnov and Krebs 1974, Parker and Courtney 1984) on the grounds that they do not adequately account for critical effects of temporal and spatial environmental heterogeneity (Crump 1981, Bernardo 1996). However, Lloyd's (1987) model concluded that modification of offspring size in response to varying resources can be adaptive even if the optimal size remains under stabilizing selection within the population.

$H$. convergens is a widely distributed Nearctic coccinellid that occurs in wide-ranging habitats from temperate to fully tropical regions. High Plains populations of H. convergens in the United States typically have two generations per year with intervening periods of aestivation in summer and hibernation in winter, unless cool summer weather permits additional aphid outbreaks (Michaud and Qureshi 2006). The first generation matures in spring, feeding on cereal aphids that bloom on flushing cool-season grasses and cereals such as winter wheat, and the second in fall when lower temperatures permit another bloom of aphids on maturing warm-season grasses and senescing broad leaf plants. Consequently, most females reproduce in only a single aphid outbreak in their life and typically do not breed in the same aphid population cycle that supported their development. Our objective in the current study was to examine lifetime trajectories of $\mathrm{RE}$ in $H$. convergens females (daily changes in fecundity, egg size, and fertility) and test for effects of a developmental constraint (larval food supply). First, we hypothesized that restriction of larval food supply would impact female reproductive allocation via effects on maternal body size and result in reductions in both egg size and egg number. Second, we hypothesized that larval food limitation would diminish the extent to which females achieved ideal schedules of RE, that is, high fecundity early in adult life.

\section{Materials and Methods}

Insect Colony. A colony of $H$. convergens was established from adult beetles collected from cultivated sunflower, Helianthus annuus L., in Hays, KS, in June, 2009. Insects were held in a growth chamber with a photoperiod of 16:8 (L:D) h length at a temperature of $24 \pm 1^{\circ} \mathrm{C}$. Adult females were isolated in plastic petri dishes ( $5.5 \mathrm{~cm}$ diameter) and were fed a diet of frozen eggs of the flour moth, Ephestia kuehniella Zeller, with water provided on a small cube of sponge, both re- freshed every day. Eggs of E. kuehniella support both development and reproduction in $H$. convergens and thus constitute an 'essential' (Hodek 1996) or 'adequate' (Michaud 2005) food. Eggs were collected daily from the inner surfaces of the petri dishes by transferring the beetles to new dishes. Eclosing larvae were reared on frozen eggs of E. kuehniella in petri dishes (as above), five per dish, to produce the next generation of adults. Insects for the experiment were obtained from this second generation of adults.

Experimental Design. The experiment was conducted under the same physical conditions used for rearing the beetle colony. Neonate larvae $(n=100)$, obtained from second generation adults, were isolated in individual petri dishes upon eclosion and then randomly assigned to one of three different treatments, representing three different levels of food availability, for rearing through to the adult stage. Larvae were provided access to frozen eggs of E. kuehniella for various periods every day: 1) $30 \mathrm{~min}$ (50 larvae), 2) $6 \mathrm{~h}$ (25 larvae), and 3) ad libitum (25 larvae). More larvae were assigned to the $30 \mathrm{~min}$ regime because relatively high mortality was expected in that treatment.

All insects were observed daily throughout the experiment and developmental time was tallied as the number of days from eclosion to adult emergence. Upon emergence, adults were weighed on an analytical balance and then isolated in a petri dish with frozen E. kuehniella provided ad libitum and water on a sponge cube, both refreshed daily.

When adults were $7 \mathrm{~d}$ old, mating pairs were established using individuals from the same feeding treatment $(n=13,11$, and 13 pairs in each of the three treatments, respectively), checking parentage to ensure that siblings were never paired together. The preoviposition period of each female was tallied as the number of days from adult emergence until the first oviposition. Observations were made daily on the number of eggs laid, the linear dimensions of the eggs, and the fraction that subsequently hatched. Using a stage micrometer under a stereomicroscope at $50 \times$ magnification, we measured the length and width of each egg to the nearest $0.02 \mathrm{~mm}$ in a sample of eggs $(n=5)$ from each oviposition day. Egg volume was estimated based on the assumption that eggs are ellipsoidal in shape (Takakura 2004):

$$
\text { Volume }=\text { Length } * \text { width }^{2} \pi / 6
$$

During preliminary work, we measured a series of eggs of varying sizes $(n=146)$ and then weighed each on a microbalance. We then used linear regression to establish the relationship between egg volume and mass, which was highly significant $\left(F_{1,145}=1,840.39\right.$; $\left.P<0.0001 ; r^{2}=0.92\right)$ and described by the following equation:

$$
\text { Mass }=0.9171 * \text { volume }+0.0078
$$

The weighing of individual eggs was exceedingly time-consuming (because of the high sensitivity of the microbalance), so we henceforth measured eggs, calculated volumes, and converted values to egg masses using this equation Hereafter, 'egg mass' refers to the 
Table 1. Life history data (mean $\pm \mathrm{SE}$ ) for $H$. convergens females reared under three feeding regimes

\begin{tabular}{|c|c|c|c|c|c|c|}
\hline \multirow{2}{*}{ Parameter } & \multicolumn{3}{|c|}{ Daily larval feeding regime } & \multirow{2}{*}{$\mathrm{df}$} & \multirow{2}{*}{$F$} & \multirow{2}{*}{$P$} \\
\hline & $30 \mathrm{~min}$ & $6 \mathrm{~h}$ & ad libitum & & & \\
\hline Total no. females & 13 & 11 & 13 & & & \\
\hline Developmental time $(\mathrm{d})$ & $29.8 \pm 1.4 \mathrm{a}$ & $22.4 \pm 0.3 \mathrm{~b}$ & $19.4 \pm 0.2 \mathrm{~b}$ & 36 & 36.3 & $<0.001$ \\
\hline Adult fresh mass (mg) & $11.0 \pm 0.7 \mathrm{c}$ & $15.2 \pm 0.3 \mathrm{~b}$ & $18.7 \pm 0.6 \mathrm{a}$ & 36 & 42.8 & $<0.001$ \\
\hline Preoviposition period (d) & $54.1 \pm 3.9 \mathrm{a}$ & $42.8 \pm 4.0 \mathrm{ab}$ & $38.3 \pm 3.5 \mathrm{~b}$ & 36 & 4.9 & 0.01 \\
\hline Reproductive period (d) & $91.0 \pm 17.9$ & $80.1 \pm 21.1$ & $104.8 \pm 18.7$ & 26 & 0.4 & 0.66 \\
\hline Egg mass $(\mu \mathrm{g})$ & $168.0 \pm 2.2$ & $174.0 \pm 5.7$ & $178.0 \pm 3.8$ & 36 & 1.7 & 0.19 \\
\hline Fecundity (total eggs) & $221.8 \pm 49.4$ & $415.1 \pm 169.7$ & $645.3 \pm 156.8$ & 36 & 2.7 & 0.08 \\
\hline Oviposition days & $19.8 \pm 4.3$ & $26.0 \pm 9.9$ & $35.7 \pm 7.7$ & 36 & 1.2 & 0.30 \\
\hline Daily fecundity (no. eggs/oviposition day) & $10.3 \pm 0.8 b$ & $12.9 \pm 1.2 \mathrm{~b}$ & $17.1 \pm 1.4 \mathrm{a}$ & 36 & 8.6 & $<0.001$ \\
\hline Fertility (\% eggs hatching) & $51.0 \pm 5.8$ & $61.0 \pm 6.0$ & $53.0 \pm 5.8$ & 36 & 0.7 & 0.49 \\
\hline Reproductive effort $^{a}$ & $3.1 \pm 0.6$ & $4.5 \pm 1.8$ & $6.3 \pm 1.7$ & 36 & 1.3 & 0.29 \\
\hline Longevity $(\mathrm{d})$ & $143.5 \pm 16.6$ & $125.1 \pm 19.2$ & $142.2 \pm 19.7$ & 26 & 0.3 & 0.75 \\
\hline
\end{tabular}

As adults, each female was fed E. kuehniella eggs ad libitum and held with a male for the duration of her reproductive life. Means bearing the same letter were not significantly different within rows (Tukey's test; $\alpha=0.05$ ).

${ }^{a}$ Total egg mass as a proportion of initial female fresh mass $=($ fecundity*egg mass $) /$ adult mass.

mean mass of individual eggs in a clutch and 'RE' to female reproductive effort (=total mass of eggs laid by a female, either daily or over entire lifespan, divided by female fresh mass at emergence).

Statistical Analysis. Treatments were compared by one-way analysis of variance (ANOVA) using PROC GLM (SAS Institute 2008) followed by Tukey's honestly significant difference (HSD) test for pairwise differences between means. Larval survival was compared among treatments using a $\chi^{2}$ test. Linear regression was used to test the relationship between oviposition day and egg mass, daily fecundity, fertility (proportion of eggs hatching), and RE. Best fit regressions (linear or polynomial) were selected on the basis of highest $r^{2}$ and $F$ values; these equations consistently yielded the lowest values of $P$. Because the lines of best fit were linear for all regressions of reproductive day on fertility, these were compared for equality of slopes using PROC REG (SAS Institute 2008). Time series observations such as changes in egg size with female age and proportional RE with female age were analyzed using PROC MIXED for repeated measures and sphericity was tested using PROC GLM (SAS Institute 2008).

\section{Results}

Forty percent of larvae died in the 30 min feeding regime, significantly more than either the $6 \%$ observed in the $6 \mathrm{~h}$ treatment $\left(\chi^{2}=517.02 ; P<0.001\right)$ or the $12 \%$ observed in the ad libitum treatment $\left(\chi^{2}=218.18 ; P<\right.$ $0.001)$; the latter two were not significantly different from one another $\left(\chi^{2}=1.70 ; P=0.191\right)$. The three larval feeding regimes (30 min, $6 \mathrm{~h}$, and ad libitum access daily) yielded three different female body sizes (small, medium, and large, respectively) and the 30 min treatment yielded the longest developmental time (Table 1). Females from the 30 min regime also took longer to begin ovipositing than ad libitum females, with $6 \mathrm{~h}$ females intermediate. There was no effect of treatment on egg mass when female lifetime means were compared (Table 1), but linear regression with all females pooled revealed a significant and positive relationship between female fresh mass and mean egg mass $\left(F_{1,36}=4.96 ; P=0.032 ; r^{2}=0.12\right)$, and between female fresh mass and daily fecundity $\left(F_{1,36}=26.05\right.$; $\left.P<0.001 ; r^{2}=0.46\right)$. The daily fecundity of ad libitum females was greater than that of $6 \mathrm{~h}$ or $30 \mathrm{~min}$ females and there was a trend toward increasing lifetime fecundity from small to large females that was not significant because of large with-group variance. Similarly, RE trended higher with increasing female size but lifetime means did not differ significantly among treatment groups (Table 1).

We focused our attention on first $25 \mathrm{~d}$ of oviposition, not including days on which eggs were not laid. This likely represents the upper limit of reproductive activity in the field, as few females in nature can be expected to experience the longevities obtained under the ideal conditions provided adult beetles in our laboratory study. Furthermore, effects of senescence on reproductive parameters likely obscure adaptive life history traits late in adult life and data became increasingly noisy as females aged, reducing our ability to resolve treatment-specific trends.

Linear regression best described how fertility (percent eggs hatching) changed over the first $25 \mathrm{~d}$ of oviposition (30 min: $F_{1,23}=56.94, P<0.001, r^{2}=0.71$ 6 h: $F_{1,23}=0.88, P=0.358, r^{2}=0.03$; ad libitum: $F_{1,23}=30.11, P<0.001, r^{2}=0.57$; Fig. 1$)$. A test for equality of slopes revealed that treatment had a significant effect on the rate of decline in fertility over time $\left(F_{2,74}=21.86 ; P<0.001\right)$. The fertility of small females declined more rapidly than that of medium $\left(F_{1,49}=\right.$ 35.51; $P<0.001)$ or large females $\left(F_{1,49}=10.32 ; P=\right.$ 0.002 ) and that of large females decreased more rapidly than that of medium females $\left(F_{1,49}=14.40\right.$; $P<0.001)$.

The ANOVA for repeated measures of individual egg mass by oviposition day revealed a significant effect of treatment $\left(F_{2,34}=60.57 ; P<0.001\right)$, significant changes in egg mass over the course of the first 25 oviposition days $\left(F_{24,496}=64.38 ; P<0.001\right)$, and a significant interaction between treatment and oviposition day $\left(F_{48,496}=15.01 ; P<0.001\right)$. Longitudinal comparisons of means were not performed because anal- 


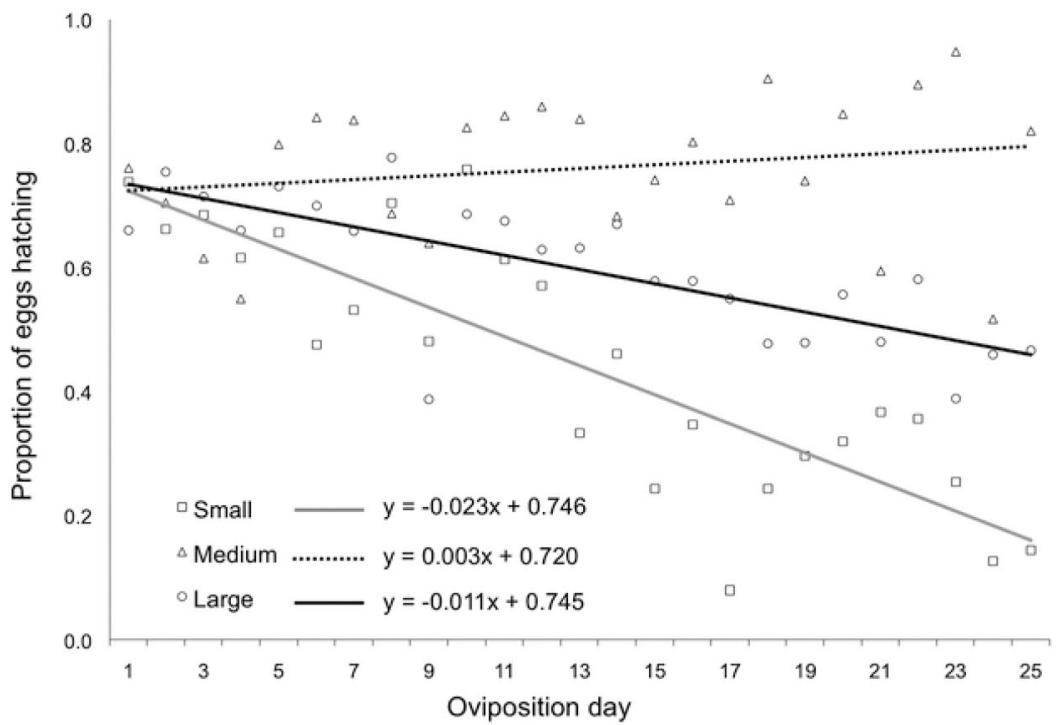

Fig. 1. Trends in mean fertility (proportion of eggs hatching) over successive oviposition days in $H$. convergens females of three different size classes produced by varying larval access to food (eggs of E. kuehniella). Line slopes were significantly different in all pairwise treatment comparisons $(P<0.001$ in all cases).

ysis of orthogonal components indicated nonhomogeneity of variance (oviposition days $1-15: F_{44,104}=141.47$; $P=0.008$ ), rendering inappropriate pair-wise comparisons that are dependent on sphericity. Changes in individual egg mass of ad libitum and $6 \mathrm{~h}$ females were best described by third order polynomial regressions $\left(F_{3,21}=\right.$ $7.80, P=0.001, r^{2}=0.53$ and $F_{3,21}=5.26, P=0.007$, $r^{2}=0.43$, respectively), whereas that of $30 \mathrm{~min}$ females was significantly linear $\left(F_{1,23}=6.41, P=0.018, r^{2}=\right.$ 0.22; Fig. 2). The mean individual egg mass of ad libitum females increased by $4.2 \%$ over the course of the first 10 oviposition days, whereas that of $6 \mathrm{~h}$ females increased by $4.5 \%$ over the first 17 . In contrast, the mean egg mass of $30 \mathrm{~min}$ females declined by $12.3 \%$ over the course of 25 oviposition days. Whereas eggs of 30 min females were $5.4 \%$ smaller than those of ad libitum females on the 10th oviposition day, they were $14.3 \%$ smaller on the 24th oviposition day.

Changes in daily fecundity over time were best described by negative second order polynomials for ad libitum and $6 \mathrm{~h}$ females $\left(F_{2,22}=4.00, P=0.003, r^{2}=\right.$ 0.27 and $F_{2,22}=1.00, P=0.383, r^{2}=0.08$, respectively), and by linear regression for small females $\left(F_{1,23}=0.75\right.$; $\left.P=0.395 ; r^{2}=0.03\right)$, although only the regression for large females was significant because of high variation within treatments (Fig. 3). Thirty minute females had

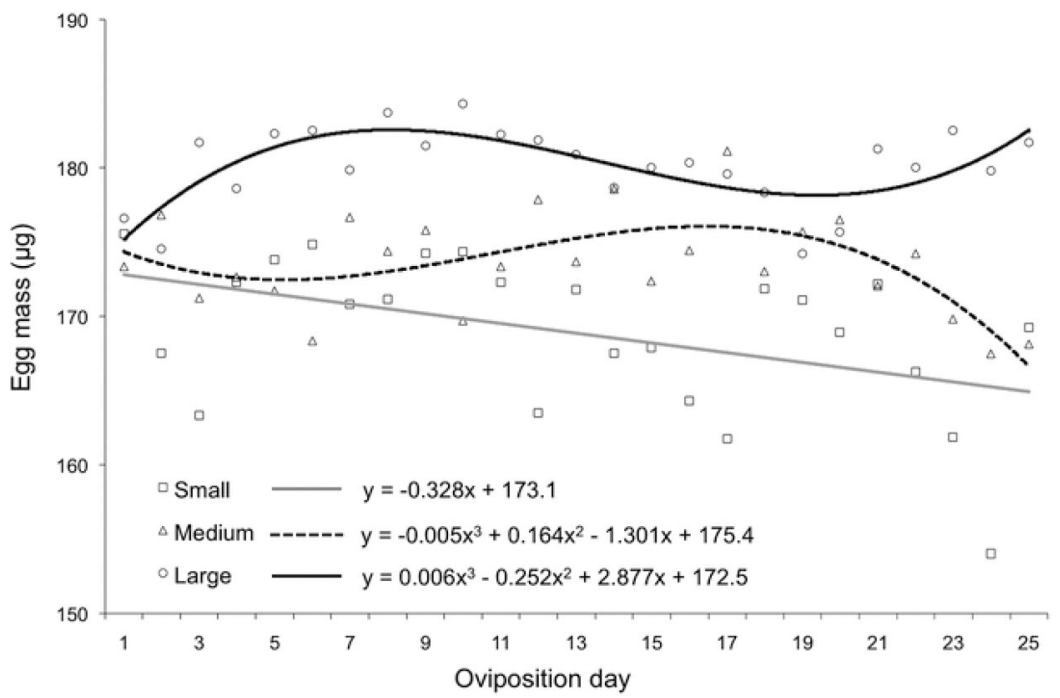

Fig. 2. Trends in mean egg mass over successive oviposition days in H. convergens females of three different size classes produced by varying larval access to food (eggs of E. kuehniella). 


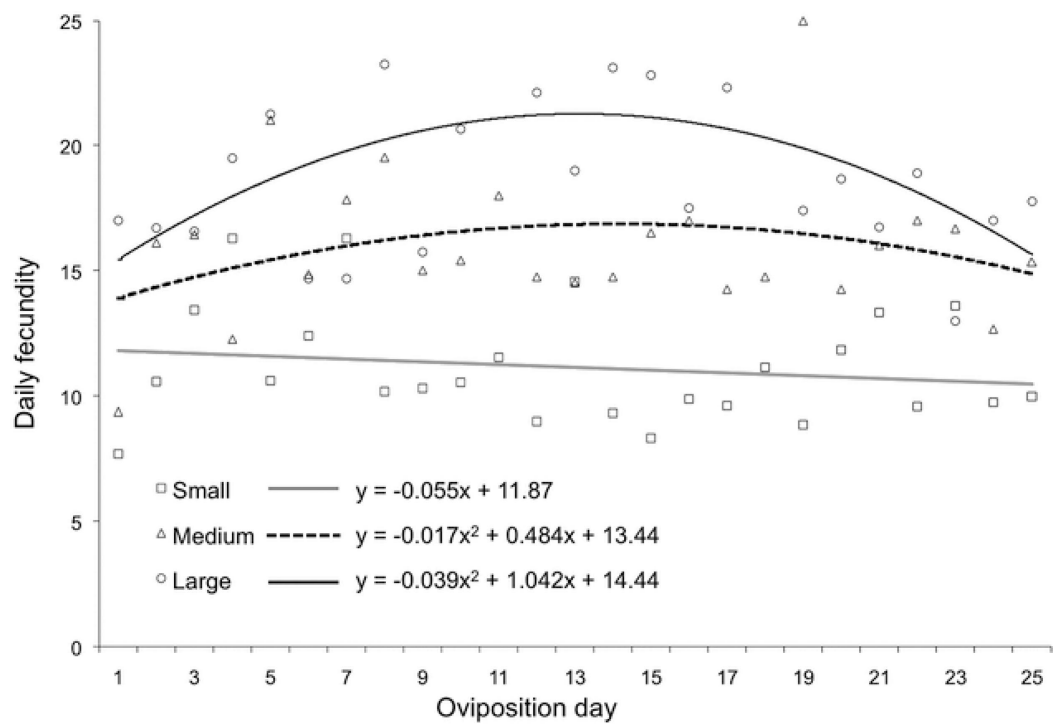

Fig. 3. Trends in mean clutch size (no. eggs per reproductive day) over successive oviposition days in H. convergens females of three different size classes produced by varying larval access to food (eggs of E. kuehniella).

lower fecundity than ad libitum females on many oviposition days (mean separations by Tukey's HSD test; $\alpha<0.05$ not shown), with $6 \mathrm{~h}$ females intermediate. Daily fecundity peaked around oviposition day 12 for ad libitum females and around day 16 for $6 \mathrm{~h}$ females.

Lifetime RE (total lifetime mass of eggs/female fresh weight at emergence) was highly variable and ranged from 0.13 to 23.24 multiples of female body mass. Trends in RE over time tended to mirror changes in daily fecundity, trending parabolic in ad libitum and $6 \mathrm{~h}$ females $\left(F_{2,22}=6.01, P=0.008, r^{2}=0.35\right.$ and $F_{2,22}=2.56$,
$P=0.09, r^{2}=0.19$, respectively) and linear in $30 \mathrm{~min}$ females $\left(F_{1,23}=2.42, P=0.133, r^{2}=0.09\right.$; Fig. 4$)$.

\section{Discussion}

The three larval feeding regimes had substantial effects on the development and subsequent reproductive activity of $H$. convergens females. An abundant larval food supply was associated with faster development, larger adult body mass, a shorter preoviposition period, larger average daily fecundity, more oviposition days, greater lifetime fecundity, and greater RE,

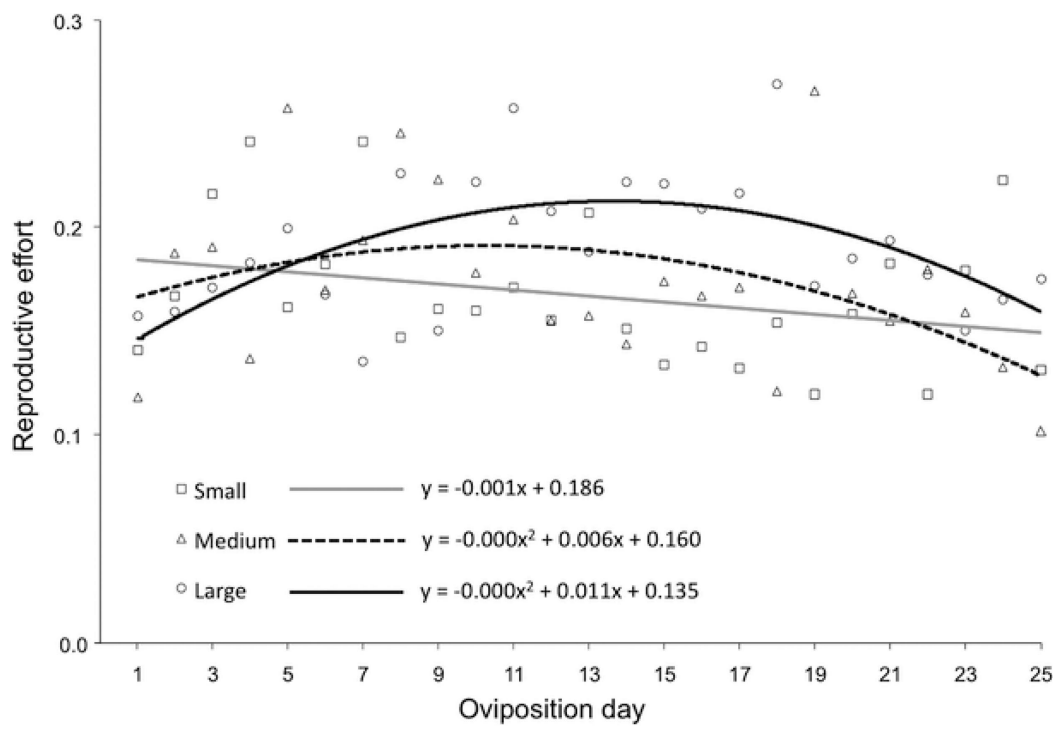

Fig. 4. Trends in mean $\mathrm{RE}(\mathrm{RE}=$ fresh mass of eggs/fresh mass of female at emergence $)$ over successive oviposition days in H. convergens females of three different size classes produced by varying larval access to food (eggs of E. kuehniella). 
although not all lifetime means were separated by the Tukey's test because of high within-group variances (Table 1). Regressions indicated significant correlations between maternal body mass and both egg mass and daily fecundity, suggesting that larger females tended to produce both larger eggs and more of them, and further supporting our primary hypothesis that both egg size and number would be negatively impacted by larval food deprivation. A positive correlation between female body size and fecundity is common across a broad range of taxa (Roff 1992, Kajita and Evans 2010), and in insects specifically (Honěk 1993). However, our results challenge earlier assumptions that coccinellid females should use surplus resources to produce more eggs of the same size (Stewart et al. 1991, Dixon and Guo 1993, Dixon 2000). Although coccinellids are primarily income-breeders (sensu Jönsson 1997) and depend largely on current food intake to support egg production (Dixon and Agarwala 2002, Honěk et al. 2008), the capital accumulated by females during development influenced not only mean fecundity and fertility, but also the dynamics of these reproductive parameters over time.

Whereas medium-sized females (fed for $6 \mathrm{~h}$ daily as larvae) sustained relatively high fertility over the course of 25 oviposition days, small females (fed for 30 min daily as larvae) exhibited a steep decline, while the fertility of large females (fed ad libitum as larvae) declined more gradually (Fig. 1). Some fertility decline may be attributable to either sperm depletion or male aging over the course of the experiment, because the seminal fluids of male insects often contain hormonal and nutritional contributions to female reproductive processes (Gillott 2003, Avila et al. 2011). Thus, a portion of all treatment effects may, in fact, be attributable to paternal effects, because males and females received the same larval feeding regimes. Whether maternally or paternally mediated, the declines in fertility in small and large females appear to reflect physiological constraints associated with extremes of body size. Thus, medium females exhibited the highest fertility over the critical first 25 ovipositions, possibly reflecting some action of stabilizing selection on body size.

The observed trajectories of RE in $H$. convergens females likely reflect the products of selection interacting with physiological constraints. Females fed ad libitum as larvae exhibited steep increases in both egg size and daily fecundity early in reproductive life that were not matched by females fed for $6 \mathrm{~h}$ (Figs. 2, 3). Reproduction early in life generally contributes more to maternal fitness than reproduction later because of the risk of adult mortality and the tendency for fertility to diminish with age. However, there are additional advantages when progeny must complete development on a time-limited resource. The time frame for oviposition in an aphid outbreak is short (Kindlmann and Dixon 1993) and selects for triangular fecundity functions that skew a larger proportion of $\mathrm{RE}$ earlier in adult life (Dixon and Agarwala 2002, Michaud and Qureshi 2006). The large females in our study appeared better able to achieve this pattern of 'front- loaded' RE than medium females, whereas small females were physiologically constrained and unable to do so, supporting our second hypothesis that larval food restriction would negatively impact reproductive schedules.

The ability of females to vary offspring size will be adaptive when the resources available for offspring fluctuate over time frames shorter than the duration of the female reproductive period (Fox and Czesak 2000, Benton et al. 2005, Benton et al. 2008). The 'hierarchy effect' posited by Parker and Begon (1986) suggests that the production of large clutches of small eggs will be favored when competition for resources is low, such as during the exponential growth stage of an aphid outbreak. Our results are also consistent with predictions of Kindsvater et al. (2010) who considered both age and maternal condition as factors affecting phenotypic variation in offspring size. Without density-dependent mortality among progeny in this model, age-dependent variation in offspring size did not evolve; 'high state' (=large) females simply produced more eggs. However, even weak density-dependent mortality leads to a strategy of increasing both offspring size and clutch size with maternal age, the effect being most pronounced in high state females. Similarly, our results revealed periods during which egg size and egg number increased in parallel in large females; however, physiological state inevitably declines as a function of age, so these trends are eventually reversed.

When conditions for offspring become unfavorable and juvenile mortality is high, females may increase egg size if this serves to improve the likelihood of progeny survival. Such conditions include environmental stress, resource depletion, increasing competition (whether between sibs, conspecifics, or heterospecifics), and an elevated risk of predation for small neonates. For example, acidic habitats are stressful for immature development of the moor frog, Rana arvalis Nilsson, (Räsänen et al. 2008) and females lay larger eggs in low $\mathrm{pH}$ environments because these presumably benefit from a lower surface area-volume ratio (Räsänen, Laurila, and Merila 2005). Parichy and Kaplan (1992) demonstrated that small tadpoles of the frog Bombina orientalis took longer to develop and metamorphosed at smaller body sizes than those of larger initial size when competing with conspecifics for a limited food supply, although these disadvantages disappeared when ad libitum food was available. Similarly, Einum and Fleming (1999) found that differences in the growth and survival of brown trout hatching from different sized eggs were only evident in poorer growth environments with some degree of competition, leading them to conclude that optimum egg size will vary across gradients of environmental quality. Sinervo (1990) experimentally reduced egg size in the lizard Sceloporus occidentalis and found that the resulting smaller juveniles had lower sprint speeds that would presumably increase their risk of predation. In insects, Fox and Mousseau (1998) showed that egg size had a marked effect on juvenile survivorship in the seed bruchid, Stator limbatus (Horn), but only 
when forced to feed on seeds of an inferior host plant. Thus, there is evidence from multiple taxa that egg size plasticity is adaptive when offspring produced at different times or in different habitats face differential survival probabilities.

Although an increase in egg size with maternal age is common in Crustacea and other taxa, it is unusual in holometabolous insects (e.g., Giron and Casas 2003) and has not been previously reported in Coleoptera (Fox and Czesak 2000). Egg size variation in insects tends to be associated with variable environmental quality (Fox and Mousseau 1998); individuals hatching from larger eggs tend to have higher survival, faster development, and often reach a larger adult size. Fischer et al. (2011) concluded that plasticity in offspring size should be favored when females can reliably predict environmental conditions for their offspring. In analogous studies with soil mites, Plaistow et al. (2007) demonstrated that as females aged, they increased progeny size at the expense of their number, especially in high food environments that permitted greater female longevity. This was construed to be adaptive in the context of the increasingly asymmetric competition facing offspring produced later in the reproductive cycle that were forced to compete with larger siblings produced earlier. For aphidophagous coccinellids, an aphid outbreak presents an initially favorable environment that deteriorates rapidly during the collapse phase; food becomes scarce, competition for resources intensifies and risks of cannibalism and intraguild predation increase, all factors that should favor an increase in egg size (Parker and Begon 1986).

Laboratory confinement likely affected females in our experiment in various ways that extended their reproductive periods and adult lives, including protection from mortality, ad libitum food provision, and forced conservation of energy that would normally be expended in dispersal and foraging. Future studies could manipulate factors such as energy expenditure in nonreproductive activities and adult food availability to determine how these factors could modify the observed trajectories of RE. For example, oosorption has recently been demonstrated in some coccinellid species (Osawa 2005, Kajita and Evans 2009) and is presumably a tactic for conserving $\mathrm{RE}$ for future opportunities when conditions for reproduction deteriorate and its consequences for egg size dynamics have not been explored. Egg size is a maternal effect (Fox and Mousseau 1998) and other, more cryptic, maternal effects might be revealed by experiments that examine progeny life histories as a function of maternal age.

\section{Acknowledgments}

The authors are grateful to Mary E. Strong, Xiaoli Wu, Brandon Boccia, and Brian Nechols for technical assistance, and to Ted Evans for reviewing an early draft of the manuscript. This work was made possible, in part, by financial support for the first author by the Colombian Sugarcane Research Center (CENICAÑA), the Fulbright Program, the Colombian Institute for the Development of Science and
Technology (COLCIENCIAS), and the National Planning Department of Colombia (DNP). Voucher specimens of $H$. convergens are deposited at the Prairie Museum of Arthropod Research in Manhattan, KS, under Lot Number 217. This is contribution 12-088-J of the Kansas State Agricultural Experiment Station.

\section{References Cited}

Avila, F. W., L. K. Sirot, B. A. LaFlamme, C. D. Rubinstein, and M. F. Wolfner. 2011. Insect seminal fluid proteins: identification and function. Annu. Rev. Entomol. 56: 2140.

Bell, G., and V. Koufopanou. 1986. The costs of reproduction, pp. 83-131. In R. Dawkins and M. Ridley (eds.), Oxford Surv. Evol. Biol., Vol. 3. Oxford University Press, Oxford, United Kingdom.

Benton, T. G., S. J. Plaistow, A. P. Beckerman, C. T. Lapsley, and S. Littlejohns. 2005. Changes in maternal investment in eggs can affect population dynamics. Proc. Roy. Soc. Lon. Ser. B. 272: 1351-1356.

Benton, T. G., J.J.H. St. Clair, and S. J. Plaistow. 2008. Maternal effects mediated by maternal age: from life histories to population dynamics. J. Anim. Ecol. 77: 1038-1046.

Bernardo, J. 1996. The particular maternal effect of propagule size, especially egg size: patterns, models, quality of evidence and interpretations. Am. Zool. 36: 216-236.

Borges, I., A. O. Soares, and J.-L. Hemptinne. 2006. Abundance and spatial distribution of aphids and scales select for different life histories in their ladybird beetle predators. J. Appl. Entomol. 130: 356-359.

Brockelman, W. Y. 1975. Competition, the fitness of offspring, and optimal clutch size. Am. Nat. 109: 677-699.

Carriere, Y., and D. A. Roff. 1995. The evolution of offspring size and number: a test of the Smith-Fretwell model in three species of crickets. Oecologia 102: 389-396.

Charnov, E. L., and J. R. Krebs. 1974. On clutch size and fitness. Ibis 116: 217-219.

Crump, M. L. 1981. Variation in propagule size as a function of environmental uncertainty for tree frogs. Am. Nat. 117: 724-737.

Dixon, A.F.G. 2000. Insect predator-prey dynamics: ladybird beetles and biological control. Cambridge University Press, Cambridge, MA.

Dixon, A.F.G., and B. K. Agarwala. 2002. Triangular fecundity function and ageing in ladybird beetles. Ecol. Entomol. 27: 433-440.

Dixon, A.F.G., and Y. Guo. 1993. Egg and cluster size in ladybird beetles (Coleoptera: Coccinellidae): the direct and indirect effects of aphid abundance. Eur. J. Entomol. 90: $457-463$.

Dixon, A.F.G., and P. Kindlmann. 2001. Role of ageing and temperature in shaping reaction norms and fecundity functions in insects. J. Evol. Biol. 14: 835-840.

Einum, S., and I. A. Fleming. 2000. Maternal effects of egg size in brown trout (Salmo truta): norms of reaction to environmental quality. Proc. Roy. Soc. Lond. Ser. B. 266: 2095-2100.

Fischer, B., B. Taborsky, and H. Kokko. 2011. How to balance the offspring quality-quantity tradeoff when environmental cues are unreliable. Oikos 120: 258-270.

Fox, C. W., and M. E. Czesak. 2000. Evolutionary ecology of progeny size in arthropods. Annu. Rev. Entomol. 45: 341369.

Fox, C. W., and T. H. Mousseau. 1998. Maternal effects as adaptations for transgenerational phenotypic plasticity in insects, pp. 159-177. In T. H. Mousseau and C. W. Fox 
(eds.), Maternal Effects as Adaptations. Oxford University Press, Oxford, United Kingdom.

Gillott, C. 2003. Male accessory gland secretions: modulators of female reproductive physiology and behavior. Annu. Rev. Entomol. 48: 163-184.

Giron, D., and J. Casas. 2003. Mothers reduce egg provisioning with age. Ecol. Lett. 6: 273-277.

Hodek, I. 1996. Food relationships, pp. 143-238. In I. Hodek and A. Honek (eds.), Ecology of Coccinellidae. Kluwer Academic, Dordrecht, The Netherlands.

Honěk, A. 1993. Intraspecific variation in body size and fecundity in insects: a general relationship. Oikos 66: 483492.

Honěk, A., A.F.G. Dixon, and M. Zdenka. 2008. Body size, reproductive allocation, and maximum reproductive rate of two species of aphidophagous Coccinellidae exploiting the same resource. Entomol. Exp. Appl. 127: 1-9.

Jönsson, K. I. 1997. Capital and income breeding as alternative tactics of resource use in reproduction. Oikos 78: 57-66.

Kajita, Y., and E. W. Evans. 2009. Ovarian dynamics and oosorption in two species of predatory lady beetles (Coleoptera: Coccinellidae). Physiol. Entomol. 34: 185-194.

Kajita, Y., and E. W. Evans. 2010. Relationships of body size, fecundity, and invasion success among predatory lady beetles (Coleoptera: Coccinellidae) inhabiting alfalfa fields. Ann. Entomol. Soc. Am. 103: 750-756.

Kindlmann, P., and A.F.G. Dixon. 1993. Optimal foraging in ladybird beetles (Coleoptera: Coccinelidae) and its consequences in their use in biological control. Eur. J. Entomol. 90: 443-450.

Kindlmann, P., and A.F.G. Dixon. 2010. Modeling population dynamics of aphids and their natural enemies, pp. 1-20. In P. Kindlmann, A.F.G. Dixon, and J. P. Michaud (eds.), Aphid Biodiversity Under Environmental Change. Springer, Berlin, Germany.

Kindsvater, H. V., S. H. Alonzo, M. Mangel, and M. B. Bonsall. 2010. Effects of age- and state-dependent allocation on offspring size and number. Evol. Ecol. Res. 12: 327-346.

Lloyd, D. G. 1987. Selection of offspring size at independence and other size-versus-number strategies. Am. Nat. 129: $800-817$.

Michaud, J. P. 2005. On the assessment of prey suitability in aphidophagous Coccinellidae. Eur. J. Entomol. 102: 385390.

Michaud, J. P., and J. A. Qureshi. 2006. Reproductive diapause in Hippodamia convergens (Coleoptera: Coccinellidae) and its life history consequences. Biol. Control 39: 193-200.

Osawa, N. 2005. The effect of prey availability on ovarian development and oosorption in the ladybird beetle Har- monia axyridis (Coleoptera: Coccinellidae). Eur. J. Entomol. 102: 503-511.

Parichy, D. M., and R. H. Kaplan. 1992. Maternal effects on offspring growth and development depend on environmental quality in the frog Bombina orientalis. Oecologia 91: 579-586.

Parker, G. A., and M. Begon. 1986. Optimal egg size and clutch size: effects of environment and maternal phenotype. Am. Nat. 128: 573-592

Parker, G. A., and S. P. Courtney. 1984. Models of clutch size in insect oviposition. Theor. Pop. Biol. 26: 27-48.

Plaistow, S. J., J.J.H. St. Clair, J. Grant, and T. G. Benton. 2007. How to put all your eggs in one basket: empirical patterns of offspring provisioning throughout a mother's lifetime. Am. Nat. 170: 520-529.

Räsänen, K., A. Laurila, and J. Merila. 2005. Maternal investment in egg size: environment- and population-specific effects on offspring performance. Oecologia 142 $546-553$.

Räsänen, K., F. Soderman, A. Laurila, and J. Merila. 2008. Geographic variation in maternal investment: acidity affects egg size and fecundity in Rana arvalis. Ecology 89: 2553-2562.

Roff, D. A. 1992. The evolution of life histories: theory and analysis. Chapman \& Hall, New York, NY.

SAS Institute. 2008. SAS software version 9.2. SAS Institute, Cary, NC.

Sinervo, B. 1990. The evolution of maternal investment in lizards: an experimental and comparative analysis. Evolution 44: 279-294.

Smith, C. C., and S. D. Fretwell. 1974. The optimal balance between size and number of offspring. Am. Nat. 108 499-506.

Stearns, S. C. 1989. Trade-offs in life-history evolution. Func. Ecol. 3: 259-268.

Stearns, S. C. 1992. The evolution of life histories. Oxford University Press, Oxford, United Kingdom.

Stewart, L. A., A.F.G. Dixon, Z. Ruzicka, and G. Iperti. 1991. Clutch and egg size in ladybird beetles. Entomophaga 36: 329-333.

Takakura, K. 2004. Variation in egg size within and among generations of the bean weevil, Bruchidius dorsalis (Coleoptera, Bruchidae): effects of host plant quality and paternal nutritional investment. Ann. Entomol. Soc. Am. 97: 346-352.

Williams, G. C. 1966. Adaptation and natural selection. Princeton University Press, Pinceton, NJ.

Received 23 January 2012; accepted 31 August 2012. 